C2008 IEEE. Personal use of this material is permitted. However, permission to reprint/republish this material for advertising or promotional purposes or for creating new collective works for resale or redistribution to servers or lists, or to reuse any copyrighted component of this work in other works must be obtained from the IEEE. 


\title{
Overview of a Media Convergence Centre $\left(\mathrm{MC}^{2}\right)$
}

\author{
Alex Talevski, Elizabeth Chang, Fellow, IEEE and Tharam Dillon, Fellow, IEEE
}

\author{
Digital Ecosystems and Business Intelligence Institute (DEBII), \\ Curtin University of Technology, GPO Box U1987, Perth, Australia 6845 \\ e-mail: (Alex.Talevski, Elizabeth.Chang, Tharam.Dillon)@cbs.curtin.edu.au
}

\begin{abstract}
Organizational alliances are rapidly being formed as a means for effective cooperation with a common goal within a targeted value chain. The combination of such communication, coordination and cooperation leads to new organisational forms and scenarios within the Digital Ecosystem space that require technological support. Convergence refers to the move towards the use of a single united interaction medium and media. Such a solution enables telecommunications services that are concurrently coupled with enterprise and internet data. Due to the versatile nature of today's extended enterprise, a flexible, feature-rich, adaptive and widely accessible converged solution is required.

This paper proposes a Media Convergence Centre $\left(\mathbf{M C}^{2}\right)$ solution that allows users to participate in a converged multimedia collaboration network using a variety of interaction devices in an easy and convenient manner.
\end{abstract}

\section{INTRODUCTION}

Extended enterprises are rapidly being formed as a collaborative approach within a targeted value chain. The combination of such communication, coordination and cooperation leads to new organisational forms and scenarios within the Digital Ecosystem space that require technological support.

Current enterprise applications have mostly provided distributed mechanisms for information sharing while telephones serve almost exclusively as communication devices $[1,2]$. Currently, enterprises utilize a heterogeneous mixture of:

- Data and voice networks (wired and wireless) - Data and voice networks are used to perform daily tasks. Typically, Public Switched Telephone Network (PSTN), Global System for Mobile (GSM) and Internet Protocol (IP) networks provide a direct communication medium and access to data services.

- Access devices - Wireless and wired telephones and computing devices are used to provide links to data and voice networks. Computing devices and software access data repositories and networks.

- Data repositories and applications - Data repositories and applications store and process enterprise information and provide optimized interfaces and views of this data.

Convergence refers to the move towards the use of a single united interaction medium and media as opposed to the many that we use today [3]. Such a solution would enable communications services that are concurrently coupled with enterprise and internet data. Data and telecom- munications convergence promises a wide range of possible solutions that will increase productivity, flexibility, reduce costs, and provide new opportunities and revenues for extended enterprises. However, such converged telecommunications and data services have been largely isolated to static environments where fixed Personal Computers (PC) and network connections are used in conjunction with customized software tools that simulate pseudo converged sessions. Generally, data presented on the internet and in enterprise applications is not available on voice networks and devices and vice-versa. Due to the diverse nature of this environment, a feature-rich, flexible and widely accessible solution is required.

Section 2 provides the Media Convergence Centre $\left(\mathrm{MC}^{2}\right)$ background. Section 3 outlines our development approach. Section 4 details our $\mathrm{MC}^{2}$ proposal. Section 4 concludes the paper.

\section{SOLUTION BACKGROUND}

\section{A. Computer Telephony Integration}

Telephone and computer systems are two technologies that impact many aspects of our daily lives. These technologies drive the world's economy and are central to the operation of virtually every enterprise. Computer Telephony Integration (CTI) is defined as the integration between computers and telephony systems [1]. CTI is a bridge that connects features of computers such as data handling, media processing and graphical user interface with telephone features such as call management and routing. Currently CTI is predominantly used to drive software-based Private Automatic Branch eXchanges (PABX). Voice over Internet Protocol (VoIP) and Interactive Voice Response (IVR) systems are essential CTI solutions.

\section{B. Voice over Internet Protocol}

Voice over Internet Protocol (VoIP) also termed IP Telephony is a solution that is commonly used by people as an alternative medium to carry-out telephone calls [4]. Generally, VoIP refers to the transport of voice traffic over a data network. VoIP hardware and software acts as an Internet transmission medium for telephone calls and other converged data [5]. Using VoIP, carrier grade voice communication is digitized and routed in discrete IP packets through a broadband connection. Telephone calls can be transmitted with little or no loss in functionality, reliability, or voice quality. VoIP is particularly useful when there 
is limited or financially prohibitive access to alternative telephony networks.

\section{Interactive Voice Response Telecom}

Interactive Voice Response Telecom (IVR) systems provide computer controlled telephone answering and routing functions, as well as facilities for the collection and provision of information. Interactive menus allow callers to input data using the telephone keypad and/or simple voice prompts. IVR devices route calls to the appropriate place or data, based on user defined steps, commands and responses to prompts. These systems may use a mixture of human and computer interaction which is provided live or pre recorded by an attendee or digitally synthesized sound to convey data to the caller. Unfortunately, industry has failed to make the most of CTI, VoIP and IVR technologies. Current use of these technologies has been limited to purely telephony applications like telephone conversations and conferencing. Existing systems rarely provide an integrated approach where more than data provision or gathering services are provided. An approach that integrates the best features of CTI, VoIP, and IVR technologies is required.

\section{Service Oriented Architecture (SOA)}

Service-Oriented Architecture (SOA) [6] embraces an architectural approach to developing composite applications from prefabricated, loosely coupled and collaborating agents. Recent research highlights the importance of SOAs in solving software complexity and integration issues [6]. SOAs encourage larger applications to be divided into smaller discrete modules. These fine-grained modules are used to produce course grained services that can be easily integrated by others. SOA defines how those service interfaces are located, executed, managed, monitored and secured. Application developers are able to browse services, select those of interest, and assemble them to create the desired functionality. Theoretically, developers can easily map distinct business processes as services that can be chained together in order to realize certain collaborative behaviour. Using SOAs dynamic composition, integration and tailoring of converged media services is possible [7]. Unfortunately, such connections require intricate knowledge of and changes to the application source code.

\section{E. Reconfigurable Software}

Research has shown that component-based software engineering leads to software that exhibits higher quality, shorter time-to-market and, therefore, lowers development cost $[8,9,10]$. However, when developing a software application by statically integrating components at implementation time it is difficult to modify the system after it has been deployed. Re-configuration, addition, removal or replacement of components may require significant changes to the application source-code. Such changes have proven to be error-prone, time consuming and expensive.

\section{F. Media Convergence}

Telecommunications and data convergence is required as a consequence of the increased flexibility that businesses demand [11]. Converged services in a single session enable users to interact in a media-rich virtual collaboration environment. Interaction is more pleasing, meaningful, effective and efficient. A media convergence centre solution would provide all industries and consumers with a new, innovative and powerful means for data rich collaboration and interaction. From an enterprise perspective, converged telecommunications and data services for its employees, partners and end customers is essential. This research stays at the forefront of such development. As a result, converged voice and data services have rapidly emerged as a popular alternative to existing telephony networks. Many sources [12, 13, 14, and 15] indicate that converged voice and data networks will grow from approximately 100,000 US households in 2004 to more than 12 million by 2009 . This proposed solution forms the foundation for more powerful features surrounding the convergence of voice, data and other interaction mediums. A media convergence solution must have the following key properties;

- Core Telecommunications Features - Private Automatic Branch eXchanges (PABX) that facilitate call management and routing.

- $\quad$ Core Computing Features - Graphical User Interfaces (GUI) promote visualization and user interaction via images, sounds, animations, video, graphs, tables, interfaces and Human Interface Devices (HID). Software applications, services and networks simplify daily tasks and, aid automation, data handling, processing and communication.

- Accessibility - Traditionally, enterprise applications allow users to access corporate data in a static location using a personal computer. However, today's business professionals are frequently on the move. As more sophisticated wireless mobile devices emerge, enterprises have to consider the growing population of mobile users that would benefit from the next generation of services [16]. Accessibility of the proposed feature-rich converged services via a variety of devices in the context of a single converged session offers enterprises great power and flexibility.

- $\quad$ Feature Rich Services - Current mobile computing devices have powerful hardware, utilize large graphical interfaces and offer network connectivity using a number of standards. With these features, the demand for media and function rich services and multi-way communication on the move will rise dramatically. A novel solution that provides access to enterprise business applications, the internet and communication services is required. This proposed solution combines major telecommunication functions such as teleconferencing, switchboards, message banks and IVR with services that access enterprise and internet data such as databases, applications and web services. These 
feature-rich services will allow users to perform tasks they would normally do while sitting at their desk. Greater efficiency, precision and faster response to market drivers then becomes possible.

- Concurrent Multi-Interface - Desired content must be delivered direct to devices in a number of formats. Voice, video and data interfaces project service data and system/user interaction. System interaction is performed through the device interface (DTMF, keyboard / mouse, touchscreen, pen etc) and/or through voice driven commands. Speech recognition and biometrics [17] aid user interaction. Being able to visualize a concept with the support of images, graphs, tables, procedures and videos while communicating over the telephone greatly enhances interaction. It enables the user to couple the power of the computer (and computing devices) with the convenience of the telephone.

- Flexible and Adaptive Environment - Due to the diverse nature of this environment, a feature-rich, flexible and widely accessible approach is required [11]. A problem faced in developing a system such as the one discussed is the complexity of service integration and reconfiguration that occurs on the different layers of telecommunications and computer systems and networks. The ability of a system to adapt or be adapted to changes in environment is referred to as its flexibility [18]. Flexible solutions are needed due to the requirement to be deployed in diverse roles and environments (its diversity) and to be flexible to unclear, lacking and/or evolving information regarding these roles and environment (uncertainty) [18]. In order to develop a flexible solution that exhibits the required solution properties, a re-configurable componentbased software model must be employed. Reconfigurable software architectures promote simplified software evolution in complex environments. Dynamic addition, removal or replacement of software components and their configuration is possible without source code discovery and modification (referred to as software tailoring). This architecture promotes an open and distributed development environment that offers an evolving service repository. Using this development approach, software quality is improved while reducing development time and cost. Software construction, customisation, integration and evolution are also simplified $[8,9,10]$.

\section{DEVELOPMENT APPROACH}

The $\mathrm{MC}^{2}$ aims to promote media collaboration using a variety of software and hardware communication devices and networks anywhere and at any time. $\mathrm{MC}^{2}$ must enable individuals and teams to interact in an easy and convenient manner. The proposal focuses an open extensible architecture that uses a thin client approach to allow system access via a range of devices and connections with no specialized software. In order to satisfy the key properties outlined earlier, we propose the following features;

\section{Core Telecommunications Features}

- Full Public Branch eXchange (PBX) services

- Telephone calling functions

- Teleconferencing features

- Call forwarding

- Message bank

- Simple Message Service (SMS)

- Call history

- Contacts repository

\section{Core Computing Features}

- Graphical User Interface (GUI)

- Internet

- eMail

- Voice over Internet Protocol (VoIP)

- Enterprise applications

- Instant Messaging (IM) including presence services

- Interactive Voice Response Telecom (IVR)

- Global Positioning System (GPS) including location services

\section{Accessibility}

- The proposed system may be employed to access a variety of telecommunications and computer services with or without the use of a personal computer or an internet connection. Interaction available via various access devices (TV, PC, PDA, telephone, mobile phone, web and others) both wired and wireless.

- Voice / data transportation mediums (IP, Wi-Fi, Bluetooth, GPS, GPRS, UMTS etc) both wired and wireless using any carrier, device and network.

- Voice over Internet Protocol (VoIP) including a variety of transportation protocols and encodings (H323, SIP, IAX etc).

- Public Switched Telephone Network (PSTN) and the Plain Old Telephone Service (POTS) services.

- No dependence on specialized hardware devices.

\section{Feature Rich Services}

- Features of computers such as data handling, media processing and graphical user interface coupled with telephone features such as call management and routing.

- Full telecommunications and teleconferencing features coupled with whiteboarding, file sharing, instant messaging, meeting and presentation management features are used to enhance collaboration.

- Customised switchboard intelligent call forwarding and voice mail features are used to manage calls as required.

- Access to enterprise and internet data

- Stored profiles

- Call history 
- Contacts, favourites and organisational information

- Voice mail

Multi Interface

- Concurrent streaming media (voice, video, web and data) where interaction can be performed using web, voice, sms, video, data and instant messaging interfaces.

- Automatic Speech Recognition (ASR)

- Dual Tone Multi Frequency (DTMF)

- Biometrics

- Transparent voice / data switchover

Flexible and Adaptive Environment

- Proven open technologies with a focus on wide compatibility.

- Re-configurable component-based framework which constitutes the skeletal support that is used as the basis for constructing the solution.

- Simplified software construction, customisation, integration and evolution.

- Complimenting components that can operate as a composite or individually.

- Flexible access to telecommunications services and enterprise and internet data.

- Allows prompt awareness and response to enterprise triggers

\section{Media Convergence Centre}

Convergence Centre ( $\mathrm{MC}^{2}$ ) (Fig 1) allows users to participate in a converged multimedia collaboration network using a variety of interaction devices in an easy and convenient manner. A thin client approach is adopted to allow access via a range of devices and connections with no specialized software. Calls, conferences and data services are provided over wired and wireless telephony and data networks. Interaction can be performed using voice, sms, video, data and instant messaging.

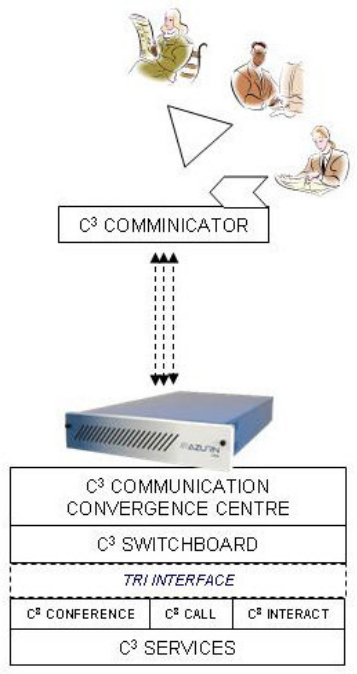

Fig. 1 Media Convergence Centre $\left(\mathrm{MC}^{2}\right)$

\section{A. $M C^{2}$ Communicator}

The $\mathrm{MC}^{2}$ Communicator (Figure 2) is central to the proposed solution. It is a full featured online communicator that is available on any browser with no additional software or hardware. It is intended to provide ad-hoc and structured collaboration as required. The $\mathrm{MC}^{2}$ Communicator is able to access all of the features of the $\mathrm{MC}^{2}$ platform. The solution gives single click access telephony, conferencing, video interaction, instant messaging, SMS and all other $\mathrm{MC}^{2}$ services. As a result of its innovative approach this interaction is available anywhere and at any time using virtually any device. Using the web client users can configure their call forwarding on the move. Detailed interaction history is stored. The approach illustrated in below is adopted in order to promote a thin client solution with wide compatibility.

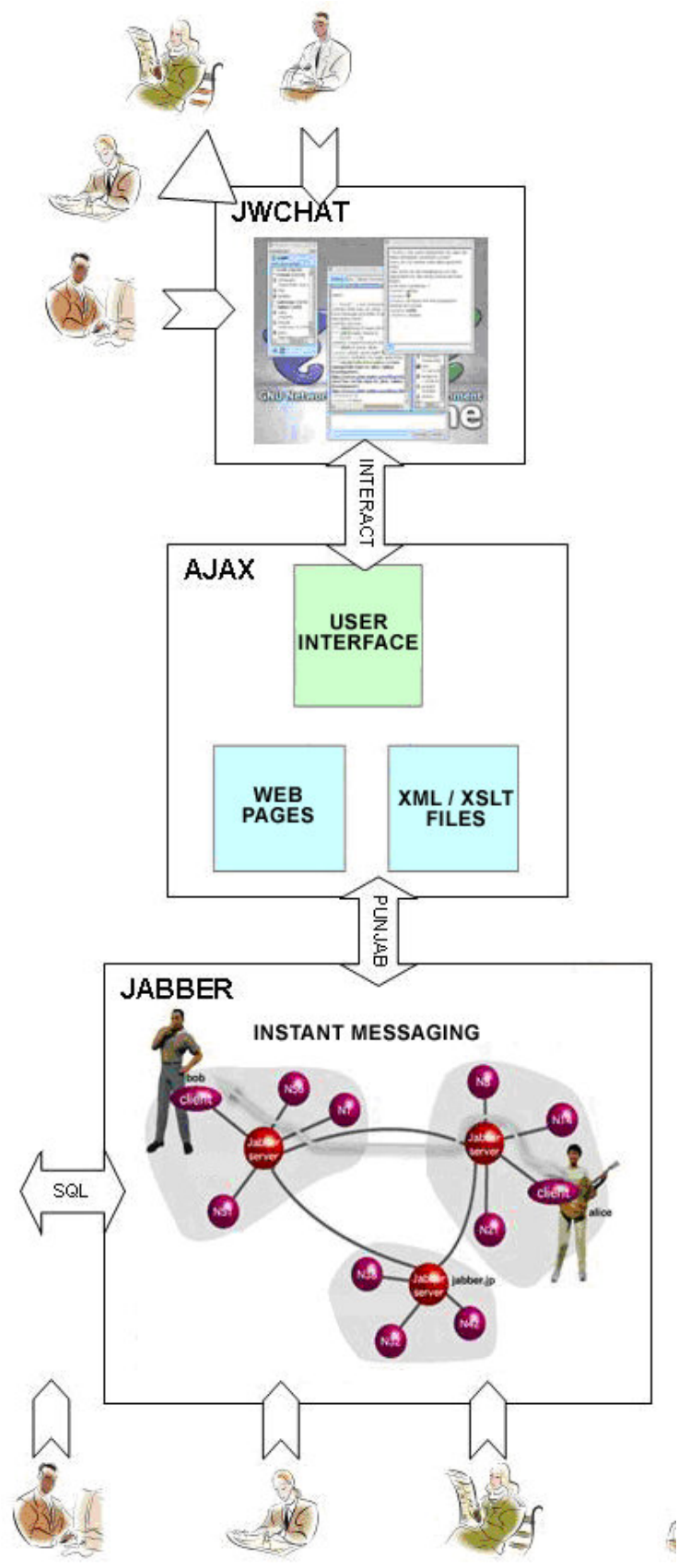

Fig. $2 \mathrm{MC}^{2}$ Communicator 
2008 Second IEEE International Conference on Digital Ecosystems and Technologies (IEEE DEST 2008) (c) 2008 IEEE.

\section{B. $M C^{2}$ Switchboard}

The $\mathrm{MC}^{2}$ Switchboard is a web-based tool for managing phone calls. It contains traditional switchboard properties and many new features that enhance switchboard functionality.

\section{C. $M C^{2}$ Conference}

The $\mathrm{MC}^{2}$ Conference (Figure 3) utilizes a thin-client approach. It provides an integrated web conferencing suite. PSTN, GSM and VoIP are integrated in a single session. The solution gives the ability to view and control the status of all participants in a conference. It is possible to interact using voice, video and data. In particular, presentation sharing and flow control as well as file sharing (with version control) are available to enhance the conference experience.

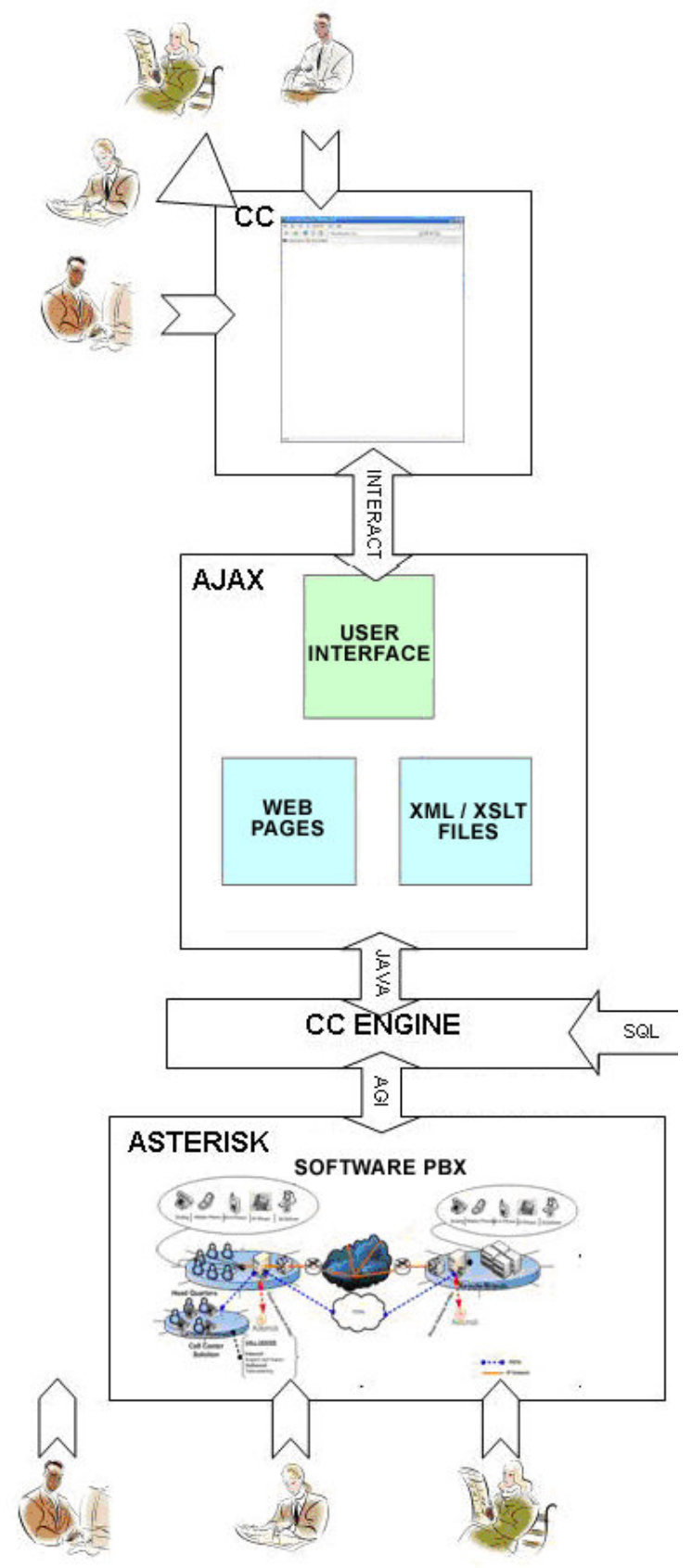

Fig. $3 \mathrm{MC}^{2}$ Conference

\section{D. $M C^{2}$ Call}

Full telephony services as per Public Switched Telephone Network (PSTN) and Plain Old Telephone Service (POTS). $\mathrm{MC}^{2}$ Call is available on any browser or telephony device.

\section{E. $M C^{2}$ Interact}

The $\mathrm{MC}^{2}$ Interact (Figure 4) solution is a fully reconfigurable Interactive Voice Response Telecom (IVR) that provides selected computer controlled telephone answering functions, collection of information and interactive menus for callers to use to input data using the telephone keypad or voice prompts. Based on user defined steps, commands and responses to prompts, calls are routed to a configured place or data. Users can create, change and remove multiple $\mathrm{MC}^{2}$ Interact maps. Figure 4 illustrates a high level architecture of the $\mathrm{MC}^{2}$ Voice Access to Data (VAD) [19] solution which provides access to enterprise and internet data The plugin services used here interact with the user using voice prompts and/or a web interface and access other components, services and repositories as required. Sample vEmail, vFinance, vStocks, vWeather, and vNews plugin services are illustrated.

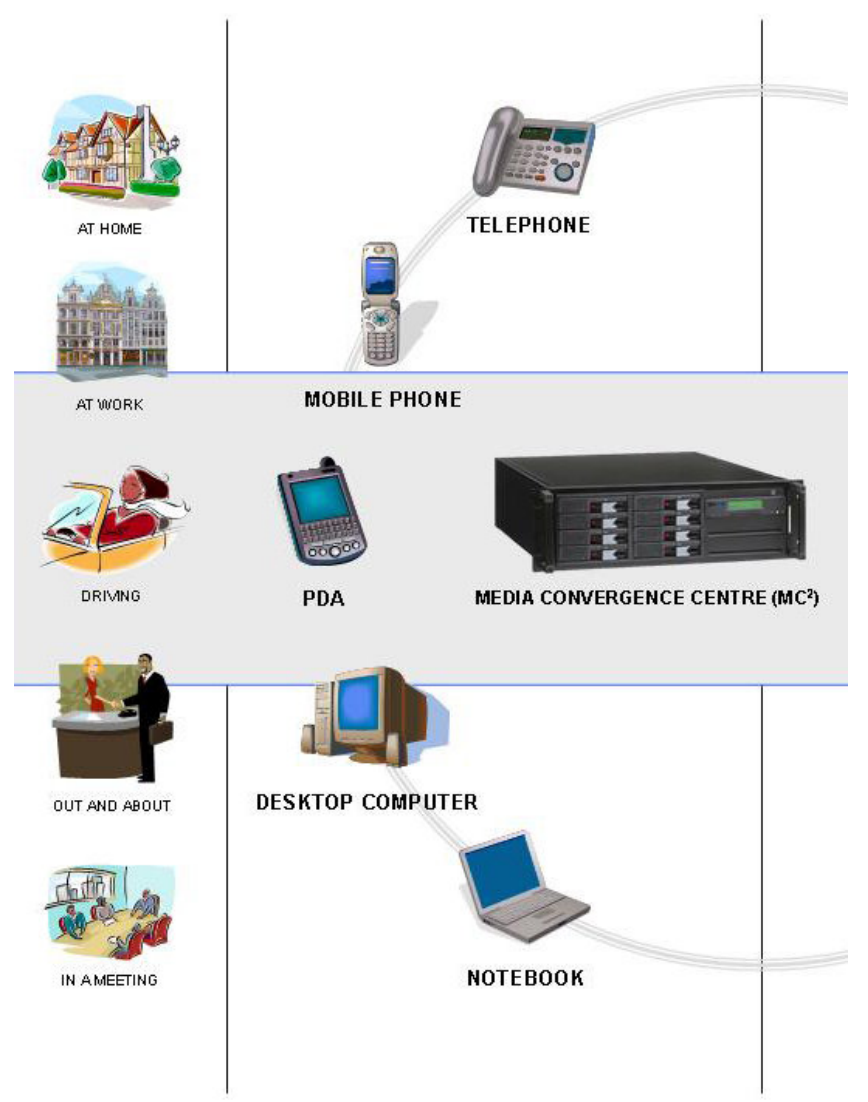

Fig. $4 \mathrm{MC}^{2}$ Interact

\section{F. $M C^{2}$ Services}

Traditionally, enterprise applications allow users to access corporate data in a static location using a personal computer. However, today's business professionals have a busy schedule and they are frequently on the move. The 
$\mathrm{MC}^{2}$ Service system (Figure 5) eliminates traditional barriers by offering a new and novel solution that allows access to a range of voice driven data services with virtually any telephony device and connection. Using a voice interface, the solution can be employed to instantly access emails, evaluate stock exchange values, check the weather, hear the news, and many more without the use of a personal computer or an internet connection. A web interface is provided to personalize and optimize the solution.

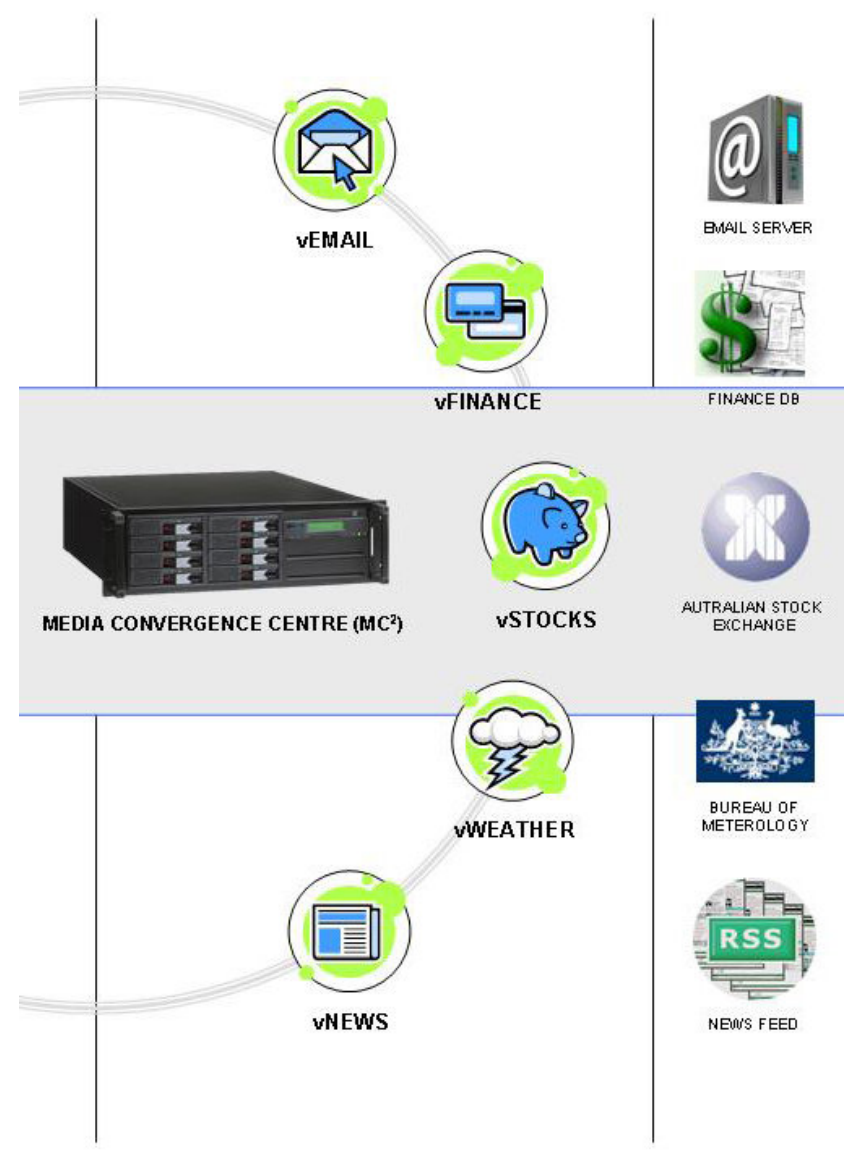

Fig. $5 \mathrm{MC}^{2}$ Services.

\section{Proposed Implementation}

An N-Tier distributed platform and component based computing architecture is proposed using the following technologies and tools;

- Asterisk - Asterisk [20] is an open source software PBX that can be programmed to create custom applications. Our system uses the java-based Application Gateway Interface (AGI) to trigger custom classes that handle incoming connections.

- Java - Java provides a platform independent environment that has wide support. Java [21] was used to interface the tools mentioned and implement the solutions in this system. Enterprise Java Beans are a suitable for the development of distributed and heterogeneous component systems. These technologies were proposed because the Asterisk AGI has wide Java support and many existing libraries and frameworks that interface the soft-switch functions of Asterisk.

- $\quad$ AT\&T TTS - The AT\&T Text-To-Speech (TTS) [22] engine generates high quality synthesized voice from text. It is integrated through the Asterisk AGI interface. Using the AT\&T TTS is it possible to adopt different dialogue files to simulate accents from different nationalities.

- Speex - This product [23] provides AutomaticSpeech-Recognition (ASR) functions. Asterisk's extended scripting commands make use of Speex to take voice commands from a user.

- Hibernate - Hibernate [24] is a high performance object / relational mapping service for Java. It uses interfaces that have defined via mapping documents to convert between the Object Oriented (OO) to the Relational Database Management Systems (RDBMS). MySQL - In order to persist hibernate objects we us the MySQL RDBMS [25].

\section{Conclusion}

From an enterprise perspective, converged voice and data services and interaction mediums to its employees, partners and end customers is essential. Converged telecommunication and, enterprise and internet services via a variety of devices in the context of a single converged session offer extended enterprises great power. The ability to visualize a concept via images, graphs, tables, procedures while communicating over the telephone greatly enhances interaction. Interaction is more pleasing, meaningful, effective and efficient. Feature-rich services allow for actions to be taken on the move with greater precision and faster response to market drivers.

This paper proposes a Media Convergence Centre $\left(\mathrm{MC}^{2}\right)$ solution that allows users to participate in a converged multimedia collaboration network using a variety of interaction devices in an easy and convenient manner.

\section{REFERENCES}

[1] C. R. Strathmeyer, "An Introduction to Computer Telephony", IEEE Communications Magazine, 35(5), May 1996, pp. 106-11.

[2] B. Benner, "Computer Telephony Integration (CTI) Industry", On-line at: http://faculty.ed.umuc.edu/ meinkej/inss690/benner/CTIpa per.htm (2006).

[3] R. Darlington, "What is multimedia convergence and why is it so important", On-line at: http://www.rogerdarlington.co.uk/Multimediaconvergence. html (2007).

[4] M. C. Hui, H.S. Matthews, "Comparative analysis of traditional telephone and Voice-over-Internet Protocol (VoIP) systems", In Proceedings of the IEEE International Symposium on Electronics and the Environment, 2004

[5] B. Goode, "Voice over Internet protocol (VoIP)", Proceedings of the IEEE, Sept. 2002 
[6] "Service-oriented architecture (SOA)", On-line at: http://www.service-architecture.com/webservices/articles/service-oriented_architecture_soa definition.html (2003).

[7] A. Talevski, E. Chang, T.S. Dillon, 'Re-Configurable Web Services for Extended Logistics Enterprise', IEEE Transaction on Industrial Informatics, vol. 1, no. 2, May 2005, pp. 74-84.

[8] M. Aoyama, "New Age of Software Development: How Component-Based Software Engineering Changes the way of Software Development", In Proceedings of the International Workshop on Component-Based Engineering, Kyoto, Japan, April 1998.

[9] L. Williams, C. Smith, "Performance Evaluation of Software Architectures", In Proceedings of the Workshop on Software and Performance, Santa Fe, New Mexico, October 1998.

[10] D. Rine, N. Nada, "Software Reuse Reference Model: Development and Validation", In Proceedings of the International Conference on Software Reuse, Victoria, Canada, June 1998.

[11] F. Bronsard, D. Bryan, W. Kozaczynski, E. Liongosari, J. Ning, A. Olafsson, J. Wetterstrand, "Toward Software Plug-and-Play", In Proceedings of the Symposium on Software Reusability, Boston, Massachusetts, May 1997.

[12] S. Phil, F. Cary, You Don't Know Jack About VoIP, Queue, 2004, 2(6), p. 30-38.

[13] W. Stallings, Data and Computer Communications (Seventh Ed.), Pearson Educational International, 2004.
[14] Deloitte, "Getting off the Ground: Why the move to VoIP is a decision for all CXOs", On-line at: http://www.deloitte.com/dtt/research/0,1015,sid\%3D2245\&cid\% 3D64027,00.html (2004).

[15] M. Grant, "Voice Quality Monitoring for VoIP Networks", Calyptech Pty. Ltd., Melbourne, 2005.

[16] G. Mao, A. Talevski, W. Rahayu 'Voice over Internet Protocol on Mobile Devices', In Proceedings of the International Conference on Computer and Information Science, Melbourne, Australia, July 2007.

[17] B.H. Juang, "Advances and challenges in speech, audio and acoustics processing for multimedia communications" In Proceeding of the International Symposium on Signal Processing and Its Applications, Brisbane, Australia, 1999.

[18] G. Booch, Object-Oriented Analysis and Design with Applications (Third Ed.), Benjamin / Cummings, Redwood City, Calif, 2007.

[19] A. Talevski, E. Chang, 'Reconfigurable Software Architecture for Voice Access to Data Services', In Proceedings of the International Conference on Digital EcoSystems and Technologies, Cairns, Australia, 2007.

[20] "Asterisk Home", On-line at: http://www.asterisk.org/ (2006)

[21] "Java Sun Home”, On-line at: http://java.sun.com/ (2006)

[22] "AT\&T TTS", On-line at:

http://www.research.att.com/viewProject.cfm?prjID=315 (2006)

[23] "Speex Home", On-line at: http://www.speex.org/ (2006)

[24] "Hibernate Home", On-line at: http://www.hibernate.org/ (2006)

[25] "MySQL Home", On-line at: http://www.mysql.com/ (2006) 